\section{RF-103 DETERMINANTS OF SLEEP PATTERNS IN HEALTH CARE PROFESSIONALS WORKING PERMANENT NIGHT SHIFTS OR ROTATING DAY SHIFTS: A MULTIVARIATE ANALYSIS BASED ON ONE-WEEK SLEEP DIARIES AND SENSOR DATA}

1Pascal Guenel, Emilie Cordina-Duverger, Brice Faraut, Julia Brettschneider, Bärbel Finkenstädt-Rand, Damien Léger, Yiyuan Zhang, Guillen Aristizabal, René Adam, Francis Levi, Amal Attari. ${ }^{1}$ INSERM, France

\subsection{6/OEM-2021-EPI.387}

Introduction Night shift and rotating shift work schedules are associated with mismatch between sleep-wake cycle and circadian rhythms, leading to sleep disturbances and adverse health effects. The impact on health and sleep patterns of shift work schedules among health care professionals working in public hospitals in Paris has been a matter of concern.

Objectives To investigate the effect of shift work schedules on sleep patterns among healthcare professionals working either as permanent night shifters (NS) or as day shifters (DS) with rotating morning and afternoon shifts.

Methods Study participants included 95 NS and 105 DS who completed a questionnaire on work history, lifestyle, sleep disorders, and job strain. Sleep and work hours were extracted from self-completed sleep diaries during a full work week. Periods of rest or activity were determined from a chest-worn sensor (PICADO ${ }^{\circledR}$ ) in 63 NS and 77 DS, using a hidden Markov model. Determinants of sleep patterns were investigated using linear mixed models.

Results According to sleep diaries, NS had both a shorter sleep duration during work days compared to DS $(5.4 \mathrm{~h} \pm$ 1.5 vs $6.8 \mathrm{~h} \pm 1.2)$, and a greater sleep debt over the study week $(3.2 \mathrm{~h} \pm 1.9$ vs $1.4 \mathrm{~h} \pm 1.5)$. The total duration of rest periods determined from the sensor was greater than the total sleep duration, particularly among NS (8.3 h \pm 1.7$)$. In addition to shift type, older age, chronotype with morning preference, high BMI and job strain were found to be independently associated with shorter duration of sleep and rest periods.

Conclusion Shift work schedules among health care professionals may lead to sleep deprivation, which may be mitigated by taking longer periods of rest bouts, particularly in permanent NS. Time slots allowing for rest periods during work hours are important to prevent health consequences of sleep disorders in these workers.

\section{RF-104 DURATION OF NIGHT SHIFT WORK IS ASSOCIATED WITH AGE ACCELERATION IN HYPERSUSCEPTIBLE FEMALE NURSES}

${ }^{1}$ Michele Carugno, Eleonora Crespi, Vincenzo Ruggiero, Paola Monti, Valentina Bollati, Angela Cecilia Pesatori, Cristina Maggioni. ${ }^{~ U n i v e r s i t y ~ o f ~ M i l a n, ~ I t a l y ~}$

\subsection{6/OEM-2021-EPI.388}

Introduction Disruption of age-related processes seems to play a relevant role in health effects related to night shift (NS) work. We had observed a decrease between telomere length (a marker of aging) and number of years in NS in subjects employed in NS $\geq 12$ years.

Objectives We now investigate the association between NS work and biological age (BA) estimated through an epigenetic signature based on methylation of five $\mathrm{CpG}$ sites in ELOVL2, C1orf132, TRIM59, KLF14 and FHL2 (Zbieć-Piekarska et al., 2017).

Methods Forty-six female nurses employed in NS for at least two years were matched by age (30-45 years) and length of service ( $\geq 1$ year) with 51 female colleagues not working in NS. Each subject filled in a semi-structured questionnaire [including the Effort Reward Imbalance (ERI) index to assess job stress] and gave a blood sample. We applied linear regression models adjusted for BMI, ERI, and smoking habit and performed stratified analyses to investigate effect modifiers. We estimated age acceleration by regressing biological age on chronological age and taking the residuals.

Results Working in NS and number of years in NS were not associated with BA in the overall population. Overweight/ obese subjects showed an increase in age acceleration per each year in NS $[0.46(0.05 ; 0.87), p=0.030, p$ for interaction $=0.097]$ as well as subjects exposed to occupational stress (ERI >1) [0.58 (0.10; 1.06), $\mathrm{p}=0.018, \mathrm{p}$ for interaction $=0.056]$. Interestingly, we observed a higher age acceleration $[0.66(0.03 ; 1.29), \mathrm{p}=0.041]$ when considering both categories combined, even if no formal interaction was apparent.

Conclusion Although hampered by the small sample size, our findings suggest a relationship between age acceleration and number of years in NS in hypersusceptible workers, i.e. overweight/obese or exposed to occupational stress.

\section{RF-142 NIGHT WORK CHARACTERISTICS AND INCIDENCE OF CORONARY HEART DISEASE: EXPOSURE-RESPONSE RELATIONS}

'Jesper Medom Vestergaard, Annett Dalbøge, Jens Peder Ellekilde Bonde, Anne Helene Garde, Åse Marie Hansen, Johnni Hansen, Ann Dyreborg Larsen, Henrik Albert Kolstad, Mikko Härmä. 'Aarhus University Hospital, Denmark

\subsection{6/OEM-2021-EPI.389}

Introduction Increasing years of recent rotating night shift work has been associated with increasing risk of coronary heart disease among nurses.

Objective We examined the association between numbers of monthly night shifts, other quantitative night work characteristics and coronary heart disease among nurses, physicians and other health care workers with the aim to provide evidence based recommendations for night work schedules.

Methods In a register-based national cohort study with a 2007-2015 follow-up, we followed 100,593 night workers (80\% women) and 158,084 day workers (78\% women) employed at public hospitals in Denmark. We defined a night shift ( $\geq 3$ hours between 12am and 5am) and different quantitative night work characteristics (i.e., number of monthly night shifts, cumulative night shifts, years with rotating night shifts, years with any night shift and consecutive night shifts) from day by day payroll information on work hours from the Danish Working Hour Database. Outcome was first time hospital admission for coronary heart disease. At end of follow-up a subgroup of 34,432 participants reported lifestyle factors i.e. tobacco smoking, alcohol consumption, body mass index and prior regular night work.

Results During follow-up, 1203 night workers (68\% women) and 2219 day workers (66\% women) were diagnosed with 